\title{
Numerical techniques for behavior of incompressible flow in steady two-dimensional motion due to a linearly stretching of porous sheet based on radial basis functions
}

https://doi.org/10.1515/nleng-2018-0029

Received February 1, 2018; revised April 11, 2018; accepted April 21, 2018.

\begin{abstract}
In this paper the boundary layer flow of a micropolar fluid due to a linearly stretching sheet which is a nonlinear system two-point boundary value problem (BVP) on semi-infinite interval has been considered. This the sheets are included the suction and injection. We solve this problem by two different collecation approaches and compare their results with solution of other methods. The proposed approaches are equipped by the direct (DRBF) and indirect radial basis functions (IRBF). Direct approach (DRBF) is based on a differential process and indirect approach (IRBF) is based on an integration process. These methods reduce solution of the problem to solution of a system of algebraic equations. Numerical results and residual norm show that the IRBF performs better than the common DRBF, and has an acceptable accuracy and high rate of convergence of IRBF process.
\end{abstract}

Keywords: Radial basis functions; Collocation method; Direct multiquadric; Indirect inverse multiquadric; System of nonlinear ODE

MSC: 34B15, 34B40

Kourosh Parand, Department of Computer Sciences, Faculty of Mathematical Sciences, Shahid Beheshti University, G.C. Tehran, Iran,

Department of Cognitive Modeling, Institute for Cognitive and Brain Sciences, Shahid Beheshti University, G.C. Tehran, Iran E-mail: k_parand@sbu.ac.ir

Yasaman Lotfi, Department of Computer Sciences, Faculty of Mathematical Sciences, Shahid Beheshti University, G.C. Tehran, Iran, E-mail: yasaman.yasi94@gmail.com

*Corresponding Author: Jamal Amani Rad, Department of Cognitive Modeling, Institute for Cognitive and Brain Sciences, Shahid Beheshti University, G.C. Tehran, Iran, E-mail: j.amanirad@gmail.com;j_amanirad@sbu.ac.ir

\section{Introduction}

\subsection{Introduction of the problem}

In recent researches the flows of micro-polar fluids has been increasingly favored due to the occurrence of these fluids in industrial processes, such as solidification of the liquid crystals, cooling of a metallic plate in a bath, exotic lubricants extrusion of metals and polymers drawing of plastic films, production of glass and paper sheets and solution of colloidal suspension. The theory of micro-polar fluids introduced by Erington [1, 2] can display the effects of local rotary inertia and couple stresses, also it is capable of explaining the flow behavior in which the classical Newtonian fluids theory is inadequate. Extensive reviews of the theory and applications were given by Łukaszewicz [3]. In this book the mathematical aspects of micro-polar fluid flow are presented. Also Ariman et al. [4] have given an excellent review of micro-polar fluids and their applications. As the problem of stretching sheet has been of great use in engineering studies, Crane [5] first studied the flow caused by an elastic sheet whose velocity varies linearly with the distance from a fixed point on the surface. Since then many researchers have reported results on stretching sheet. The micro-polar flow on a moving flat plate was studied by Ishak et al. [6-8]. Hassanien and Gorla [9] by considering suction and injection obtained numerical solution. The same problem, using a method of successive approximations, was analyzed by Hady [10]. The Newtonian fluid counterpart was studied by Gupta and Gupta [11]. Recently, some new aspects of micro-polar fluids and their applications have been investigated by Rahman et al. [12-17]. More recently, limiting behavior of micro-polar flow due to a linearly stretching porous sheet have been investigated by $[18,19]$. In present investigation we deal with the boundary layer flow of a micro-polar fluid due to linearly stretching sheet. 


\subsection{Introduction of radial basis functions}

Radial basis functions (RBFs) interpolations are techniques for representing a function starting with data on scattered nodes. This technique first appears in the literature as a method for scattered data interpolation, and the method was highly favored after being reviewed by Franke [20], who found it to be the most impressive of the many methods he tested. Later, Kansa [21, 22] in 1990 proposed an approximate solution of linear and nonlinear differential equations (DEs) using RBFs. For the last years, the radial basis functions (RBFs) method was known as a powerful tool for the scattered data interpolation problem. The main advantage of numerical methods which use radial basis functions is the meshless characteristic of these methods. The use of radial basis functions as a meshless method for the numerical solution of ordinary differential equations (ODEs) and partial differential equations (PDEs) is based on the collocation method. Kansa's method has recently received a great deal of attention from researchers [23-32].

Recently, Kansa's method was extended to solve various ordinary and partial differential equations including the nonlinear Klein-Gordon equation [27], regularized long wave (RLW) equation [33], high order ordinary differential equations [34], the case of heat transfer equations [35], Hirota-Satsuma coupled KdV equations [36], second-order parabolic equation with nonlocal boundary conditions [37], Second-order hyperbolic telegraph equation [38], incompressible Eyring-Powell fluid over a linear stretching sheet [66], pricing of options $[67,68]$, inverse Cauchy-Stefan problem $[69,70]$, non-Fickian flows with mixing length growth in porous media [71], unsteady flow of gas in a semi-infinite porous medium [72], and so on [73]. All of the radial basis functions have global support, and in fact many of them, such as multiquadrics (MQ), do not even have isolated zeros [27, 33, 39]. The RBFs can be compactly and globally supported, infinitely differentiable, and contain a free parameter $c$, called the shape parameter [33, 39, 40]. For more basic details about RBFs the interested readers can refer to the recent books and paper by Buhmann [39, 41] and Wendland [42], compactly and globally supported; and convergence rate of the radial basis functions.

Despite many studies done to find algorithms for selecting the optimum values of $c$ [43-45], the optimal choice of shape parameter is an open problem which is still under intensive investigation. For example, Carlson and Foley [44] found that the shape parameter is problem dependent. They [44] observed that for rapidly varying functions, a small value of $c$ should be used, but a large value should be used if the function has a large curvature. Tarwater [45] found that by increasing $c$, the Root-MeanSquare (RMS) of error dropped to a minimum and then increased sharply afterwards. In general, as $c$ increases, the system of equations to be solved becomes ill-conditioned. Cheng et al. [43] showed that when $c$ is very large then the RBFs system error is of exponential convergence. But there is a certain limit for the value $c$ after which the solution breaks down. In general, as the value of the shape parameter $c$ increases, the matrix of the system to be solved becomes highly ill-conditioned and hence the condition number can be used in determining the critical value of the shape parameter for an accurate solution [43]. For some new work on optimal choice of shape parameter, we refer the interested reader to the recent work of [46].

There are two basic approaches for obtaining basis functions from RBFs, namely direct approach (DRBF) based on a differential process [22] and indirect approach (IRBF) based on an integration process [25, 34, 47]. Both approaches were tested on the solution of second order DEs and the indirect approach was found to be superior to the direct approach [25].

In contrast, the integration process is much less sensitive to noise [34, 48]. Based on this observation, it is expected that through the integration process, the approximating functions will be much smoother and therefore have higher approximation power $[34,48]$.

To numerically explore the IRBF methods with shape parameters for which the interpolation matrix is too poorly conditioned to use standard methods mentioned by the authors as ref [49]. This is perhaps the major advantage of the IRBFs as RBFs methods are typically not employed in applications using the optimal shape parameters, but using some value of the parameter safely away from the region of ill-conditioning [50].

Some of the infinitely smooth RBFs choices are listed in Table 1. The RBFs can be of various types, for example: polynomials of any chosen degree such as linear, cubic, etc., thin plate spline (TPS), multiquadrics (MQ), inverse multiquadrics (IMQ), Gaussian forms (GA), hyperbolic secant (sech) form etc. Regarding the inverse quadratic, inverse multiquadric (IMQ), hyperbolic secant (sech) and Gaussian (GA), the coefficient matrix of RBFs interpolating is positive definite and, for multiquadric (MQ), it has one positive eigenvalue and the remaining ones are all negative [51].

In this paper, we use the multiquadric (MQ) to direct approach and inverse multiquadrics (IMQ) to indirect approach radial basis functions for finding the solution of main problem. The MQ was first developed by Hardy [52] in 1971 as a multidimensional scattered interpolation 
Table 1: Some well-known radial basis functions $\left(r=\left\|x-x_{i}\right\|^{2}=\right.$ $\left.r_{i}\right), c>0$

\begin{tabular}{lc}
\hline Name of functions & Definition \\
\hline Multiquadrics (MQ) & $\sqrt{r+c^{2}}$ \\
Inverse multiquadrics (IMQ) & $1 /\left(\sqrt{r+c^{2}}\right)$ \\
Gaussian (GA) & $\exp (-c r)$ \\
Hyperbolic secant (sech) & $\operatorname{sech}(c \sqrt{r})$ \\
\hline
\end{tabular}

method in modeling of the earth gravitational field. It was not recognized by most of the academic researchers until Franke [20] published a review paper on the evaluation of two-dimensional interpolation methods, whereas MQ was ranked as the best, based on its accuracy, visual aspect, sensitivity to parameters, execution time, storage requirements, and ease of implementation.

For convenience the solution we use RBFs with collocation nodes $\left\{x_{j}\right\}_{j=1}^{N}$ which are the zeros of the shifted Legendre polynomial $L_{N}(x), 0 \leq x \leq t_{f}$. The shifted Legendre polynomials $L_{i}(x)$ are defined on the interval $\left[0, t_{f}\right]$ and satisfy the following formulae [53]:

$$
\begin{aligned}
& L_{0}(x)=1, \quad L_{1}(x)=\frac{2}{t_{f}} x-1, \\
& L_{i+1}(x)=\frac{2 i+1}{i+1}\left(\frac{2}{t_{f}} x-1\right) L_{i}(x)-\frac{i}{i+1} L_{i-1}(x), \\
& \quad i=1,2,3, \ldots .
\end{aligned}
$$

This paper is arranged as follows: in Section 2 we present a brief formulation of the problem. In Section 3, we describe the properties of radial basis functions. In Section 4 we implement the problem with direct and indirect radial basis functions methods and in Section 5 we report our numerical finding and demonstrate the accuracy of the proposed methods. The conclusions are discussed in the final Section.

\section{Problem formulation}

An incompressible micro-polar fluid in steady twodimensional motion, driven by a flat sheet that is linearly stretching away from a fixed point is assumed. Otherwise; the fluid would have been at rest. The governing boundary layer equations obtained by [18], as systems of nonlinear ordinary differential equation are given by:

$$
\begin{aligned}
& F^{\prime \prime \prime}+F F^{\prime \prime}-F^{\prime 2}=-\varepsilon H^{\prime}, \\
& H^{\prime \prime}-\beta^{2} H=\frac{1}{2} \beta^{2} F^{\prime \prime},
\end{aligned}
$$

with boundary conditions

$$
\begin{array}{ll}
F(0)=\phi, & F^{\prime}(0)=1, \quad F^{\prime}(\infty)=0, \\
H(0)=0, & H(\infty)=0,
\end{array}
$$

where prime denotes differentiation with respect to $\eta$; the similarity coordinate measuring distances normal to the sheet. $F^{\prime}, H, \varepsilon, \beta$ and $\phi$ are velocity, micro-rotation, coupling parameter, a constant characteristic of the fluid and suction velocity through the porous surface.

\section{Radial basis functions}

\subsection{Definition of radial basis functions}

Let $\mathbb{R}^{+}=\{x \in \mathbb{R}, x \geq 0\}$ be the non-negative half-line and let $\phi: \mathbb{R}^{+} \rightarrow \mathbb{R}$ be a continuous function with $\phi(0) \geq 0$. A radial basis functions on $\mathbb{R}^{d}$ is a function of the form

$$
\phi\left(\left\|X-X_{i}\right\|\right),
$$

where $X, X_{i} \in \mathbb{R}^{d}$ and $\|\cdot\|$ denotes the Euclidean distance between $X$ and $X_{i}$ s. If one chooses $N$ points $\left\{X_{i}\right\}_{i=1}^{N}$ in $\mathbb{R}^{d}$ then by custom

$$
s(X)=\sum_{i=1}^{N} \lambda_{i} \phi\left(\left\|X-X_{i}\right\|\right) ; \quad \lambda_{i} \in \mathbb{R},
$$

is called a radial basis functions as well [54].

The standard radial basis functions are categorized into two major classes [36]:

Class 1. Infinitely smooth RBFs [36, 55]:

These basis functions are infinitely differentiable and heavily depend on the shape parameter $c$ e.g. Hardy multiquadric (MQ), Gaussian(GA), inverse multiquadric (IMQ), and inverse quadric(IQ) (See Table 1).

Class 2. Infinitely smooth (except at centers) RBFs [36, 55]: The basis functions of this category are not infinitely differentiable. These basis functions are shape parameter free and have comparatively less accuracy than the basis functions discussed in the Class 1. For example, thin plate spline, etc [36].

As far as the radial basis functions are concerned, several choices are possible, such as the so-called multiquadrics, inverse multiquadrics, Gaussian RBFs (see, for example, [41]). In particular, the multiquadrics, the inverse multiquadrics and the Gaussian RBFs contain a free shape parameter on which the performances of the RBF approximation strongly depend. Precisely, values of the shape parameters that yield a high spatial resolution (i.e. a high level of accuracy) also lead to severely ill-conditioned 
linear systems. Therefore, one has to find a value of the shape parameter such that the numerical results are satisfactorily accurate but at the same time the overall approximation do not blow up (due to ill-conditioning problems). Now, in the technical literature, various approaches for selecting the RBF shape parameter have been proposed, see, e.g., $[20,43,44,46,59-63]$. These algorithms, which are often based on rules of thumbs and on semi-analytical relations, can yield satisfactory results in some circumstances. Nevertheless, to the best of our knowledge, a method to choose the RBF shape parameter which is rigorously established and is proven to perform well in the general case is still lacking.

\subsection{RBFs interpolation}

The one dimensional function $y(x)$ to be interpolated or approximated can be represented by an RBFs as:

$$
y(x) \approx y_{N}(x)=\sum_{i=1}^{N} \lambda_{i} \phi_{i}(x)=\Phi^{T}(x) \Lambda,
$$

where

$$
\begin{aligned}
& \phi_{i}(x)=\varphi\left(\left\|x-x_{i}\right\|\right), \\
& \Phi^{T}(x)=\left[\phi_{1}(x), \phi_{2}(x), \ldots, \phi_{N}(x)\right], \\
& \Lambda=\left[\lambda_{1}, \lambda_{2}, \ldots, \lambda_{N}\right]^{T},
\end{aligned}
$$

$x$ is the input and $\left\{\lambda_{i}\right\}_{i=1}^{N}$ are the set of coefficients to be determined. By choosing $N$ interpolate nodes $\left\{x_{i}\right\}_{i=1}^{N}$, we can approximate the function $y(x)$.

$$
y_{j}=\sum_{i=1}^{N} \lambda_{i} \phi_{i}\left(x_{j}\right), \quad j=1,2, \ldots, N .
$$

To summarize discussion on coefficient matrix, we define:

$$
\mathbf{A} \Lambda=\mathbf{Y},
$$

where

$$
\begin{aligned}
\mathbf{y} & =\left[y_{1}, y_{2}, \ldots, y_{N}\right]^{T}, \\
\mathbf{A} & =\left[\Phi^{T}\left(x_{1}\right), \Phi^{T}\left(x_{2}\right), \ldots, \Phi^{T}\left(x_{N}\right)\right]^{T}, \\
& =\left[\begin{array}{cccc}
\phi_{1}\left(x_{1}\right) & \phi_{2}\left(x_{1}\right) & \ldots & \phi_{N}\left(x_{1}\right) \\
\phi_{1}\left(x_{2}\right) & \phi_{2}\left(x_{2}\right) & \ldots & \phi_{N}\left(x_{2}\right) \\
\vdots & \vdots & \ddots & \vdots \\
\phi_{1}\left(x_{N}\right) & \phi_{2}\left(x_{N}\right) & \ldots & \phi_{N}\left(x_{N}\right)
\end{array}\right] .
\end{aligned}
$$

Note that $\phi_{i}\left(x_{j}\right)=\varphi\left(\left\|x_{i}-x_{j}\right\|\right)$ therefore we have $\phi_{i}\left(x_{j}\right)=\phi_{j}\left(x_{i}\right)$ consequently $\mathbf{A}=\mathbf{A}^{T}$.
All the infinitely smooth RBFs choices are listed in Table 1 will give coefficient matrices $\mathbf{A}$ in Eq. (3.3) which are symmetric and nonsingular [51], i.e. there is a unique interpolant of the form Eq. (3.1) no matter how the distinct data points are scattered in any number of space dimensions. In the cases of inverse quadratic, inverse multiquadric (IMQ), hyperbolic secant (sech) and Gaussian (GA) the matrix $\mathbf{A}$ is positive definite and, for multiquadric (MQ), it has one positive eigenvalue and the remaining ones are all negative [51].

\subsection{Direct radial basis functions (DRBF)}

In this method, we write the goal function $y(x)$ by using a linear combination of $N$ RBFs:

$$
y(x) \approx y_{N}(x)=\sum_{i=1}^{N} \lambda_{i} \phi_{i}(x)=\Phi^{T}(x) \Lambda,
$$

which $\Phi(x)$ and $\Lambda$ are intruduced in the Eq. (3.2). Now, for derivative of any order $y(x)$, we have

$$
\begin{gathered}
\frac{d^{k} y(x)}{d x^{k}}=\frac{d^{k}}{d x^{k}}\left(\sum_{i=1}^{N} \lambda_{i} \phi_{i}(x)\right)=\sum_{i=1}^{N} \lambda_{i} \frac{d^{k} \phi_{i}(x)}{d x^{k}} \\
=\sum_{i=1}^{N} \lambda_{i} E_{i}^{[k]}(x)=\left(\mathbf{E}^{[k]}(x)\right)^{T} \Lambda,
\end{gathered}
$$

where

$$
\begin{aligned}
& E_{i}^{[k]}(x)=d^{k} \phi_{i}(x) / d x^{k}, \quad k=0,1, \ldots, p, \\
& \left(\mathbf{E}^{[k]}(x)\right)^{T}=\left(E_{1}^{[k]}(x), E_{2}^{[k]}(x), \ldots, E_{N}^{[k]}(x)\right), \\
& \mathbf{E}^{[0]}(x)=\Phi(x) .
\end{aligned}
$$

Let $p$ be the highest order of the derivative under consideration the boundary value ODEs in general form:

$$
\begin{cases}F\left(x, y, y^{\prime}, \ldots, y^{(p-1)}, y^{(p)}\right)=0, & a \leq x \leq b, \\ y^{(i)}\left(e_{i}\right)=\alpha_{i+1}, & i=0,1, \ldots, p-1,\end{cases}
$$

where $e_{i} \in\{a, b\}$ and $y^{(i)}(x)=d^{i} y(x) / d x^{i}, F$ is known function and $\left\{\alpha_{i}\right\}_{i=1}^{p}$ are known constants. By substituting Eq. (3.4) and Eq. (3.5) in (3.6), we obtain the residual functions:

$$
\begin{aligned}
\operatorname{Res}(x) & =F\left(x, y, y^{\prime}, \ldots, y^{(p)}\right) \\
= & F\left(x,\left(\mathbf{E}^{[0]}(x)\right)^{T} \Lambda,\left(\mathbf{E}^{[1]}(x)\right)^{T} \Lambda, \ldots,\left(\mathbf{E}^{[p]}(x)\right)^{T} \Lambda\right) .
\end{aligned}
$$

The set of equations for obtaining the coefficients $\left\{\lambda_{i}\right\}_{i=1}^{N}$ come from equalizing Eq. (3.7) to zero at $N-p$ collocation 
nodes $\left\{x_{j}\right\}_{j=1}^{N-p}$ plus $p$ boundary conditions:

$$
\begin{cases}\operatorname{Res}\left(x_{j}\right)=0, & j=1,2, \ldots, N-p, \\ \left(\mathbf{E}^{[k]}\left(e_{i}\right)\right)^{T} \Lambda=\alpha_{i+1}, & k=0,1, \ldots, p-1 .\end{cases}
$$

\subsection{Indirect radial basis functions (IRBF)}

In this approach [34, 56-58], RBFs are used to represent the highest order derivatives $p$ of a function $y(x)$, e.g.

$$
\frac{d^{p} \hat{y}(x)}{d x^{p}}=\sum_{i=1}^{N} \hat{\lambda}_{i} \hat{\phi}_{i}(x)=\hat{\Lambda}^{T} \hat{\Phi}(x),
$$

where

$$
\begin{aligned}
& \hat{\Phi}^{T}(x)=\left[\hat{\phi}_{1}(x), \hat{\phi}_{2}(x), \ldots, \hat{\phi}_{N}(x)\right], \\
& \hat{\Lambda}=\left[\hat{\lambda}_{1}, \hat{\lambda}_{2}, \ldots, \hat{\lambda}_{N}\right]^{T} .
\end{aligned}
$$

The obtained derivative expression is then integrated to yield expressions for lower order derivatives and finally for the original function itself [34]. Let $p$ be the highest order of the derivative under consideration the boundary value ODEs in general form Eq. (3.6). We define integral operation $I$ as:

$$
I(f)=\int f(t) d t
$$

then we can define:

$$
\begin{aligned}
& \hat{y}^{(p)}(x)=\frac{d^{p} \hat{y}(x)}{d x^{p}}=\hat{\Lambda}^{T} \hat{\Phi}(x), \\
& I\left(\hat{y}^{(p)}(x)\right)=\hat{\Lambda}^{T} I(\hat{\Phi}(x))+\hat{q}^{T} \vec{d}_{p}, \\
& \hat{y}^{(p-1)}(x)=\hat{\Lambda}^{T} I(\hat{\Phi})+d_{p-1} q_{0}(x)=\hat{\Lambda}^{T} I(\hat{\Phi})+\hat{q}^{T} \vec{d}_{p-1}, \\
& \hat{y}^{(p-2)}(x)=\hat{\Lambda}^{T} I^{2}(\hat{\Phi})+d_{p-1} q_{1}(x)+d_{p-2} q_{0}(x) \\
& =\hat{\Lambda}^{T} I^{2}(\hat{\Phi})+\hat{q}^{T} \vec{d}_{p-2}, \\
& \hat{y}^{\prime}(x)=\hat{\Lambda}^{T} I^{p-1}(\hat{\Phi})+\sum_{i=0}^{p-2} q_{i}(x) d_{i+1}=\hat{\Lambda}^{T} I^{p-1}(\hat{\Phi})+\hat{q}^{T} \overrightarrow{d_{1}}, \\
& \hat{y}(x)=\hat{\Lambda}^{T} I^{p}(\hat{\Phi})+\sum_{i=0}^{p-1} q_{i}(x) d_{i}=\hat{\Lambda}^{T} I^{p}(\hat{\Phi})+\hat{q}^{T} \vec{d}_{0}, \\
& \hat{y}^{(i)}(x)=\left[I^{p-i}(\hat{\Phi}) \hat{q}^{T}\right]\left[\begin{array}{c}
\hat{\Lambda} \\
\vec{d}_{i}
\end{array}\right],
\end{aligned}
$$

where:

$$
\begin{aligned}
& \hat{y}^{(i)}\left(e_{i}\right)=\alpha_{i+1}, \quad i=0,1,2, \ldots, p-1, \\
& \hat{\Phi}^{T}=\left[\hat{\phi}_{1}(x), \hat{\phi}_{2}(x), \ldots, \hat{\phi}_{N}(x)\right], \\
& I^{i^{T}}(\hat{\Phi})=\left[I^{i}\left(\hat{\phi}_{1}(x)\right), I^{i}\left(\hat{\phi}_{2}(x)\right), \ldots, I^{i}\left(\hat{\phi}_{N}(x)\right)\right], \\
& \hat{\Lambda}^{T}=\left[\lambda_{1}, \lambda_{2}, \ldots, \lambda_{N}\right], \\
& \vec{d}_{i}^{T}=\left[\bar{d}_{i} \quad \overline{0}\right]_{1 \times p},\left(\bar{d}_{i}\right)^{T}=\left[d_{i}, \ldots, d_{p-1}\right]_{1 \times(p-i)}, \\
& (\overline{0})^{T}=[0, \ldots, 0]_{1 \times i}, i=0,1, \ldots, p-1 \\
& \hat{q}^{T}=\left[q_{0}(x), q_{1}(x), \ldots, q_{p-1}(x)\right], \\
& q_{i}(x)=\frac{x^{i}}{i !} .
\end{aligned}
$$

Now to obtain $\left\{\lambda_{i}\right\}_{i=1}^{N}$, we define the residual function:

$$
\operatorname{Res}(x)=F\left(x, \hat{y}, \hat{y}^{\prime}, \ldots, \hat{y}^{(p)}\right) .
$$

The set of equations for obtaining the coefficients $\left\{\lambda_{i}\right\}_{i=1}^{N}$ and $\left\{d_{j}\right\}_{j=0}^{p-1}$ come from equalizing Eq. (3.14) to zero at $N$ interpolate nodes $\left\{x_{j}\right\}_{j=1}^{N}$

$$
\begin{cases}\operatorname{Res}\left(x_{j}\right)=0, & j=1,2, \ldots, N, \\ \hat{y}^{(i)}\left(e_{i}\right)=\alpha_{i+1}, & i=0,1, \ldots, p-1 .\end{cases}
$$

\section{Solving the model}

In the first step of our analysis, we approximate $F(\eta)$ and $H(\eta)$ for solving the model by DRBF:

$$
\begin{aligned}
& F(\eta) \simeq y_{1}(\eta)=\sum_{i=1}^{N} \lambda_{i}^{[1]} \phi_{i}(\eta)=\Phi^{T}(x) \Lambda^{[1]}, \\
& H(\eta) \simeq y_{2}(\eta)=\sum_{i=1}^{N} \lambda_{i}^{[2]} \phi_{i}(\eta)=\Phi^{T}(x) \Lambda^{[2]} .
\end{aligned}
$$

Also, $F^{\prime \prime \prime}(\eta)$ and $H^{\prime \prime}(\eta)$ for solving the model by IRBF:

$$
\begin{aligned}
& F^{\prime \prime \prime}(\eta) \simeq \frac{d^{3}}{d \eta^{3}} \hat{y_{1}}(\eta)=\sum_{i=1}^{N} \lambda_{i}^{[1]} \phi_{i}(\eta)=\left(\hat{\Lambda}^{[1]}\right)^{T} \hat{\Phi}(x), \\
& H^{\prime \prime}(\eta) \simeq \frac{d^{2}}{d \eta^{2}} \hat{y_{2}}(\eta)=\sum_{i=1}^{N} \lambda_{i}^{[2]} \phi_{i}(\eta)=\left(\hat{\Lambda}^{[2]}\right)^{T} \hat{\Phi}(x) .
\end{aligned}
$$

The general form of problem appear to:

$$
\left\{\begin{array}{l}
R\left(\eta, F, H, F^{\prime}, H^{\prime}, F^{\prime \prime}, H^{\prime \prime}, F^{\prime \prime \prime}\right)=0, \\
R\left(\eta, F, H, F^{\prime}, H^{\prime}, F^{\prime \prime}, H^{\prime \prime}, F^{\prime \prime \prime}\right)=0, \\
F(0)=\phi, F^{\prime}(0)=1, F^{\prime}(\infty)=0, \\
H(0)=0, H(\infty)=0 .
\end{array}\right.
$$


To solve this problem we define residual functions for DRBF:

$$
\left\{\begin{array}{l}
\operatorname{Res}_{1}(\eta)=R\left(\eta, y_{1}, y_{2}, y_{1}^{\prime}, y_{2}^{\prime}, \ldots\right), \\
\operatorname{Res}_{2}(\eta)=R\left(\eta, y_{1}, y_{2}, y_{1}^{\prime}, y_{2}^{\prime}, \ldots\right),
\end{array}\right.
$$

and residual functions for IRBF:

$$
\left\{\begin{array}{l}
\operatorname{Res}_{1}(\eta)=R\left(\eta, \hat{y_{1}}, \hat{y_{2}}, \hat{y_{1}}, \hat{y_{2}}{ }^{\prime}, \ldots\right), \\
\operatorname{Res}_{2}(\eta)=R\left(\eta, \hat{y_{1}}, \hat{y_{2}}, \hat{y_{1}}, \hat{y_{2}}{ }^{\prime}, \ldots\right) .
\end{array}\right.
$$

The unknown coefficients $\left\{\lambda_{i}^{[1]}\right\}_{i=1}^{N}$ and $\left\{\lambda_{i}^{[2]}\right\}_{i=1}^{N}$ come from equalizing $\operatorname{Res}_{1}(\eta)$ and $\operatorname{Res}_{2}(\eta)$ to zero at $N$ interpolate nodes $\eta_{i}$ from zeros of the shifted Legendre polynomial (For more detail about this polynomial, please see $[64,65])$ between 0 and $\eta_{\infty}$ which we set $\eta_{\infty}=10$ for this problem.

\subsection{Solving the model by DRBF}

For obtaining the solution by DRBF approach, the residual functions are constructed by substituting Eq. (4.1) in Eq. (4.4):

$$
\begin{aligned}
\operatorname{Res}_{1}(\eta)= & \left(\mathbf{E}^{[3]}(\eta)\right)^{T} \Lambda^{[1]}+\left(\mathbf{E}^{[0]}(\eta)\right)^{T} \Lambda^{[1]}\left(\mathbf{E}^{[2]}(\eta)\right)^{T} \Lambda^{[1]} \\
& +\varepsilon\left(\mathbf{E}^{[1]}(\eta)\right)^{T} \Lambda^{[2]}, \\
\operatorname{Res}_{2}(\eta)= & \left(\mathbf{E}^{[2]}(\eta)\right)^{T} \Lambda^{[2]}-\beta^{2}\left(\mathbf{E}^{[0]}(\eta)\right)^{T} \Lambda^{[2]} \\
& -\frac{1}{2} \beta^{2}\left(\mathbf{E}^{[2]}(\eta)\right)^{T} \Lambda^{[1]} .
\end{aligned}
$$

By using $N-3$ interpolate nodes $\left\{x_{j}\right\}_{j=1}^{N-3}$ in $\operatorname{Res}_{1}(\eta)$ and $N-2$ interpolate nodes $\left\{x_{j}\right\}_{j=1}^{N-2}$ in $\operatorname{Res}_{2}(\eta)$ plus five boundary conditions $F(0)=\phi, F^{\prime}(0)=1, F^{\prime}\left(\eta_{\infty}\right)=0, H(0)=$ $0, H\left(\eta_{\infty}\right)=0$, the set of equations can be solved, consequently the coefficients $\left\{\lambda_{i}^{[1]}\right\}_{i=1}^{N}$ and $\left\{\lambda_{i}^{[2]}\right\}_{i=1}^{N}$ will be obtained:

$$
\begin{cases}\operatorname{Res}_{1}\left(x_{j}\right)=0, & j=1,2, \ldots, N-3, \\ \operatorname{Res}_{2}\left(x_{j}\right)=0, & j=1,2, \ldots, N-2, \\ \left(\mathbf{E}^{[0]}(0)\right)^{T} \Lambda^{[1]}=\phi, & \\ \left(\mathbf{E}^{[1]}(0)\right)^{T} \Lambda^{[1]}=1, & \\ \left(\mathbf{E}^{[1]}\left(\eta_{\infty}\right)\right)^{T} \Lambda^{[1]}=0, & \\ \left(\mathbf{E}^{[0]}(0)\right)^{T} \Lambda^{[2]}=0, & \\ \left(\mathbf{E}^{[0]}\left(\eta_{\infty}\right)\right)^{T} \Lambda^{[2]}=0, & \end{cases}
$$

\subsection{Solving the model by IRBF}

For obtaining the solution by IRBF approach, the residual functions are constructed by substituting Eq. (4.2) in
Eq. (4.5) and using Eq. (3.12):

$$
\begin{aligned}
& \operatorname{Res}_{1}(\eta)=\left[\begin{array}{ll}
I^{0^{T}}(\hat{\Phi}) & \hat{q}^{T}
\end{array}\right]\left[\begin{array}{c}
\hat{\Lambda}^{[1]} \\
{\overrightarrow{d_{3}}}^{[1]}
\end{array}\right]
\end{aligned}
$$

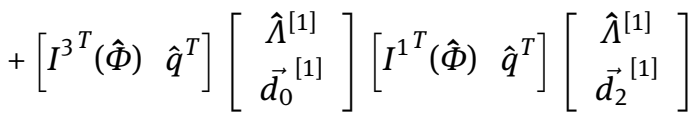

$$
\begin{aligned}
& -\left[\begin{array}{ll}
I^{2^{T}}(\hat{\Phi}) & \hat{q}^{T}
\end{array}\right]\left[\begin{array}{c}
\hat{\Lambda}^{[1]} \\
{\overrightarrow{d_{1}}}^{[1]}
\end{array}\right]\left[\begin{array}{ll}
I^{2^{T}}(\hat{\Phi}) & \hat{q}^{T}
\end{array}\right]\left[\begin{array}{c}
\hat{\Lambda}^{[1]} \\
\vec{d}_{1}^{[1]}
\end{array}\right] \\
& +\varepsilon\left[I^{1^{T}}(\hat{\Phi}) \hat{q}^{T}\right]\left[\begin{array}{c}
\hat{\Lambda}^{[2]} \\
\vec{d}_{1}^{[2]}
\end{array}\right] \\
& \operatorname{Res}_{2}(\eta)=\left[\begin{array}{ll}
I^{0^{T}}(\hat{\Phi}) & \hat{q}^{T}
\end{array}\right]\left[\begin{array}{l}
\hat{\Lambda}^{[2]} \\
{\overrightarrow{d_{2}}}^{[2]}
\end{array}\right] \\
& -\beta^{2}\left[I^{2^{T}}(\hat{\Phi}) \hat{q}^{T}\right]\left[\begin{array}{l}
\hat{\Lambda}^{[2]} \\
{\overrightarrow{d_{0}}}^{[2]}
\end{array}\right] \\
& -\frac{1}{2} \beta^{2}\left[\begin{array}{ll}
I^{1}(\hat{\Phi}) & \hat{q}^{T}
\end{array}\right]\left[\begin{array}{l}
\hat{\Lambda}^{[1]} \\
\vec{d}_{2}^{[1]}
\end{array}\right]
\end{aligned}
$$

By using $N$ interpolate nodes $\left\{x_{j}\right\}_{j=1}^{N}$ in $\operatorname{Res}_{1}(\eta)$ and $N$ interpolate nodes $\left\{x_{j}\right\}_{j=1}^{N}$ in $\operatorname{Res}_{2}(\eta)$ plus five boundary conditions of Eq. (4.3) the set of equations can be solved, consequently the coefficients matrix $\hat{\Lambda}^{[1]}, \hat{\Lambda}^{[2]}$ and coefficients $\left\{d_{i}^{[1]}\right\}_{i=1}^{3}$ and $\left\{d_{i}^{[2]}\right\}_{i=1}^{2}$ will be obtained.

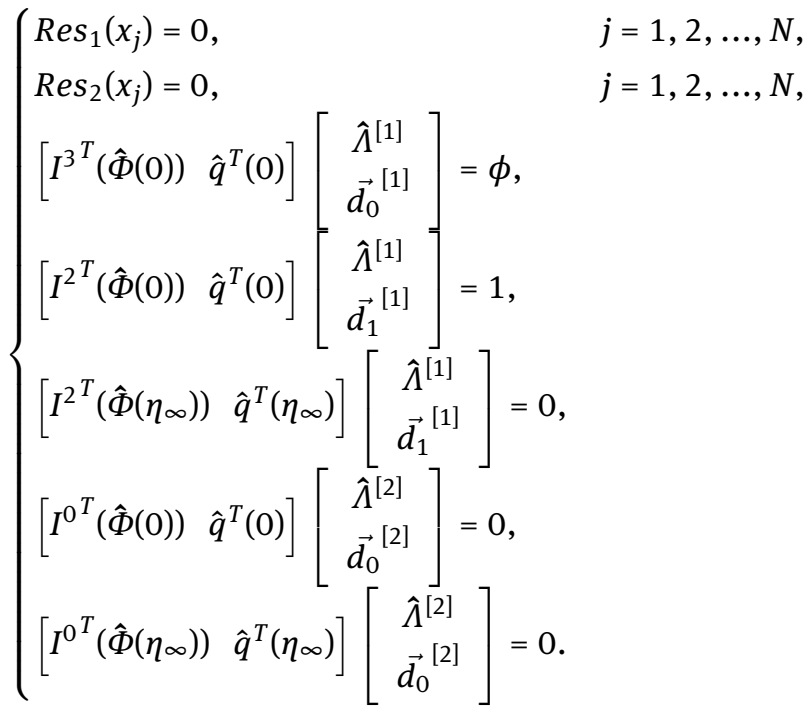

Remark: It is worth to mention that it is so difficult generally to solve the final system of nonlinear equations (Sections 4.1 and 4.2) even by Newton's method. The main difficulty with a such system is that how we can choose initial guess to handle the Newton's method, in other words, how many solutions the system of nonlinear equations admit. We think the best way to discover proper initial guess (or initial guesses) is to solve system analytically for very small $N$ (by using symbolic softwares program such as 


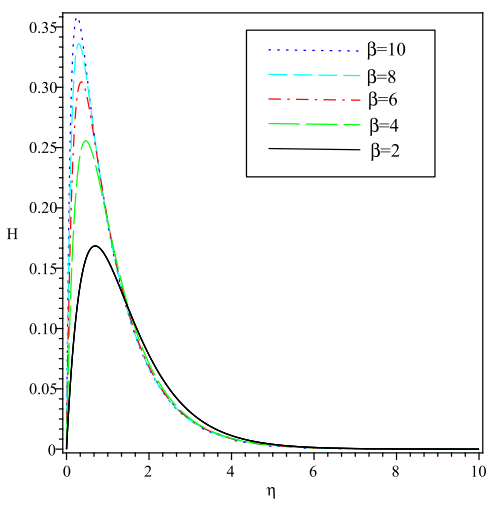

Fig. 1: The profile micro-rotation $H(\eta)$ obtained by the direct RBFs for different values of $\beta$ at $\phi=0$ and $\varepsilon=0.1$.

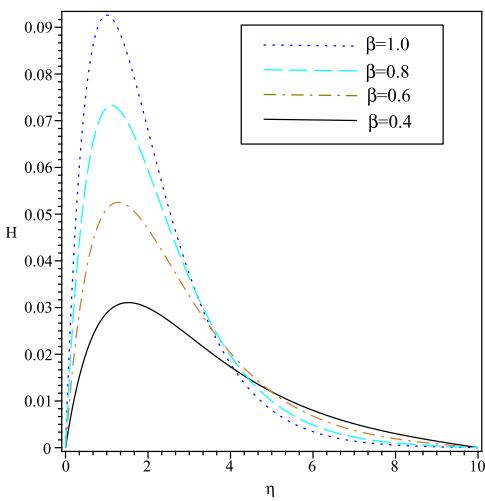

Fig. 2: The profile micro-rotation $H(\eta)$ obtained by the direct RBFs for different values of $\beta$ at $\phi=0$ and $\varepsilon=0.1$.

Mathematica or Maple) and then we can find proper initial guesses and particularly multiplicity of solutions of such system. This action has been done by starting from proper initial guesses with the maximum number of ten iterations.

\section{Numerical results and discussion}

In this section, the numerical results of the model equations presented in Section 1, obtained by using DRBF and IRBF are discussed. The numerical implementation is carried out in microsoft.maple.13, with hardware configuration: desktop 64-bit Intel Core 2 Duo CPU, 4 GB of RAM, 64-bit Operating System. For this propose and understand the effects of flow parameters, we presented Figures 1-15 and Tables 2-7.

Figures 1-2 show the effect of $\beta$ as a constant characteristic of the fluid on the $H(\eta)$ as the micro-rotation. It is observed that by increasing the value of $\beta$, micro-rotation

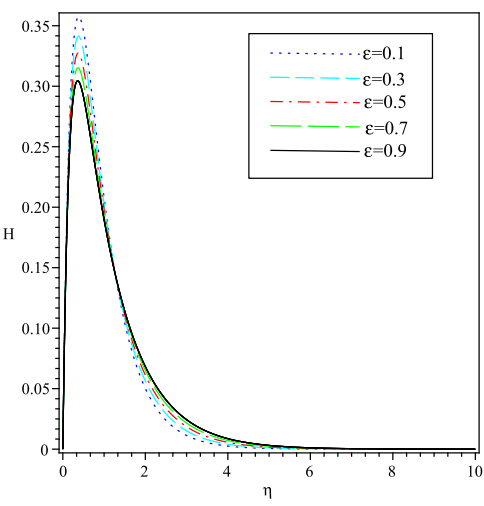

Fig. 3: The profile micro-rotation $H(\eta)$ obtained by the direct RBFs for different values of $\varepsilon$ at $\beta=6$ and $\phi=0$.

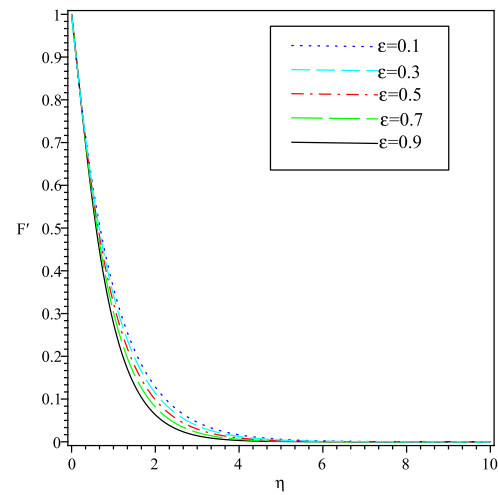

Fig. 4: The profile velocity $F^{\prime}(\eta)$ obtained by the direct RBFs for different values of $\varepsilon$ at $\beta=6$ and $\phi=0$.

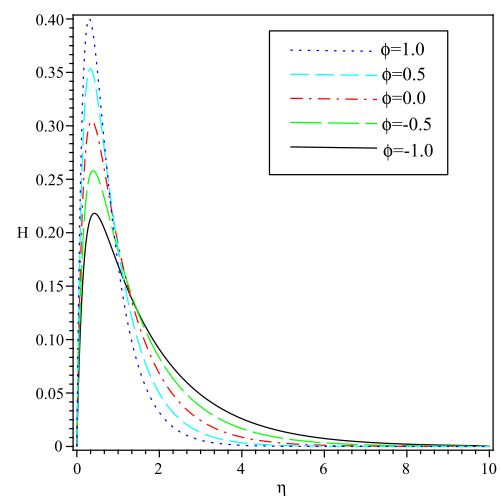

Fig. 5: The profile micro-rotation $H(\eta)$ obtained by the direct RBFs for different values of $\phi$ at $\beta=6$ and $\varepsilon=0.1$. 
Table 2: Comparison between direct RBFs and other solutions of $F^{\prime \prime}(0)$ and $H^{\prime}(0)$ for some $\beta$ at $\varepsilon=0.1$ and $\phi=0$.

\begin{tabular}{|c|c|c|c|c|c|c|c|c|}
\hline \multirow[b]{2}{*}{$\beta$} & \multicolumn{2}{|c|}{ Runge-Kutta [19] } & \multicolumn{4}{|c|}{ DRBF method } & \multicolumn{2}{|c|}{ HAM [19] } \\
\hline & $F^{\prime \prime}(0)$ & $H^{\prime}(0)$ & $N$ & c & $F^{\prime \prime}(0)$ & $H^{\prime}(0)$ & $F^{\prime \prime}(0)$ & $H^{\prime}(0)$ \\
\hline $0 . \overline{4}$ & -0.99859 & 0.05714 & 26 & 9.5 & -0.998591 & 0.057179 & -0.99875 & 0.05244 \\
\hline 0.6 & -0.99752 & 0.11268 & 26 & 9.5 & -0.997522 & 0.112683 & -0.99758 & 0.10876 \\
\hline 0.8 & -0.99644 & 0.17814 & 26 & 9.0 & -0.996442 & 0.178143 & -0.99627 & 0.17590 \\
\hline 1 & -0.99540 & 0.25059 & 26 & 9.0 & -0.995400 & 0.250587 & -0.99504 & 0.24963 \\
\hline 2 & -0.99117 & 0.66860 & 26 & 7.5 & -0.991165 & 0.668599 & -0.99071 & 0.66803 \\
\hline 4 & -0.98625 & 1.60433 & 30 & 7.4 & -0.986199 & 1.604329 & -0.98564 & 1.60245 \\
\hline 6 & -0.98358 & 2.57743 & 34 & 7.0 & -0.983487 & 2.577417 & -0.98289 & 2.57162 \\
\hline 8 & -0.98191 & 3.56272 & 36 & 6.5 & -0.981647 & 3.562724 & -0.98293 & 3.54947 \\
\hline 10 & -0.98077 & 4.55348 & 38 & 6.0 & -0.980221 & 4.553448 & -0.98458 & 4.52889 \\
\hline
\end{tabular}

Table 3: Comparison between direct RBFs and other solutions of $F^{\prime \prime}(0)$ and $H^{\prime}(0)$ for some $\varepsilon$ at $\beta=6$ and $\phi=0$.

\begin{tabular}{|c|c|c|c|c|c|c|c|c|}
\hline \multirow[b]{2}{*}{$\varepsilon$} & \multicolumn{2}{|c|}{ Runge-Kutta [19] } & \multicolumn{4}{|c|}{ DRBF method } & \multicolumn{2}{|c|}{ HAM [19] } \\
\hline & $F^{\prime \prime}(0)$ & $H^{\prime}(0)$ & $N$ & $c$ & $F^{\prime \prime}(0)$ & $H^{\prime}(0)$ & $F^{\prime \prime}(0)$ & $H^{\prime}(0)$ \\
\hline $0 . \overline{1}$ & -0.98358 & 2.57743 & 34 & 7.0 & -0.983487 & 2.577417 & -0.98290 & 2.57589 \\
\hline 0.3 & -0.94983 & 2.59083 & 34 & 7.0 & -0.949639 & 2.590684 & -0.94819 & 2.58873 \\
\hline 0.5 & -0.91482 & 2.60677 & 34 & 7.2 & -0.914617 & 2.606575 & -0.91247 & 2.60439 \\
\hline 0.7 & -0.87841 & 2.62606 & 34 & 6.2 & -0.878065 & 2.625707 & -0.87498 & 2.60688 \\
\hline 0.9 & -0.84043 & 2.64993 & 34 & 6.2 & -0.840119 & 2.649604 & -0.85964 & 2.62407 \\
\hline
\end{tabular}

Table 4: Comparison between direct RBFs and other solutions of $F^{\prime \prime}(0)$ and $H^{\prime}(0)$ for some $\phi$ at $\beta=6$ and $\varepsilon=0.1$.

\begin{tabular}{|c|c|c|c|c|c|c|c|c|}
\hline \multirow[b]{2}{*}{$\phi$} & \multicolumn{2}{|c|}{ Runge-Kutta [19] } & \multicolumn{4}{|c|}{ DRBF method } & \multicolumn{2}{|c|}{ HAM [19] } \\
\hline & $F^{\prime \prime}(0)$ & $H^{\prime}(0)$ & $N$ & $c$ & $F^{\prime \prime}(0)$ & $H^{\prime}(0)$ & $F^{\prime \prime}(0)$ & $H^{\prime}(0)$ \\
\hline 1.0 & -1.60501 & 3.85515 & 34 & 6.5 & -1.604819 & 3.855190 & -1.56517 & 3.94163 \\
\hline 0.5 & -1.26561 & 3.18526 & 34 & 6.5 & -1.265468 & 3.185293 & -1.25134 & 3.08218 \\
\hline 0.0 & -0.98358 & 2.57743 & 34 & 7.0 & -0.983487 & 2.577417 & -0.98289 & 2.57162 \\
\hline-0.5 & -0.76447 & 2.06901 & 34 & 7.2 & -0.764397 & 2.068947 & -0.77038 & 2.12820 \\
\hline-1.0 & -0.60299 & 1.67194 & 34 & 7.2 & -0.602932 & 1.671866 & -0.59347 & 1.69706 \\
\hline
\end{tabular}

Table 5: Comparison between indirect RBFs and other solutions of $F^{\prime \prime}(0)$ and $H^{\prime}(0)$ for some $\beta$ at $\varepsilon=0.1$ and $\phi=0$.

\begin{tabular}{|c|c|c|c|c|c|c|c|c|}
\hline \multirow[b]{2}{*}{$\beta$} & \multicolumn{2}{|c|}{ Runge-Kutta [19] } & \multicolumn{4}{|c|}{ IRBF method } & \multicolumn{2}{|c|}{ HAM [19] } \\
\hline & $F^{\prime \prime}(0)$ & $H^{\prime}(0)$ & $N$ & $c$ & $F^{\prime \prime}(0)$ & $H^{\prime}(0)$ & $F^{\prime \prime}(0)$ & $H^{\prime}(0)$ \\
\hline $0 . \overline{4}$ & -0.99859 & 0.05714 & 14 & 2.0 & -0.998590 & 0.057179 & -0.99875 & 0.05244 \\
\hline 0.6 & -0.99752 & 0.11268 & 14 & 2.0 & -0.997520 & 0.112682 & -0.99758 & 0.10876 \\
\hline 0.8 & -0.99644 & 0.17814 & 14 & 2.0 & -0.996439 & 0.178144 & -0.99627 & 0.17590 \\
\hline 1 & -0.99540 & 0.25059 & 14 & 2.0 & -0.995398 & 0.250591 & -0.99504 & 0.24963 \\
\hline 2 & -0.99117 & 0.66860 & 25 & 2.0 & -0.991161 & 0.668593 & -0.99071 & 0.66803 \\
\hline 4 & -0.98625 & 1.60433 & 33 & 1.0 & -0.986239 & 1.604313 & -0.98564 & 1.60245 \\
\hline 6 & -0.98358 & 2.57743 & 33 & 0.5 & -0.983591 & 2.577462 & -0.98289 & 2.57162 \\
\hline 8 & -0.98191 & 3.56272 & 35 & 0.5 & -0.981908 & 3.562717 & -0.98293 & 3.54947 \\
\hline 10 & -0.98077 & 4.55348 & 38 & 0.5 & -0.980758 & 4.553430 & -0.98458 & 4.52889 \\
\hline
\end{tabular}

Table 6: Comparison between indirect RBFs and other solutions of $F^{\prime \prime}(0)$ and $H^{\prime}(0)$ for some $\varepsilon$ at $\beta=6$ and $\phi=0$.

\begin{tabular}{|c|c|c|c|c|c|c|c|c|}
\hline \multirow[b]{2}{*}{$\varepsilon$} & \multicolumn{2}{|c|}{ Runge-Kutta [19] } & \multicolumn{4}{|c|}{ IRBF method } & \multicolumn{2}{|c|}{ HAM [19] } \\
\hline & $F^{\prime \prime}(0)$ & $H^{\prime}(0)$ & $N$ & $c$ & $F^{\prime \prime}(0)$ & $H^{\prime}(0)$ & $F^{\prime \prime}(0)$ & $H^{\prime}(0)$ \\
\hline $0 . \overline{1}$ & -0.98358 & 2.57743 & 33 & 0.5 & -0.983591 & 2.577462 & -0.98290 & 2.57589 \\
\hline 0.3 & -0.94983 & 2.59083 & 33 & 0.5 & -0.949827 & 2.590820 & -0.94819 & 2.58873 \\
\hline 0.5 & -0.91482 & 2.60677 & 33 & 0.5 & -0.914809 & 2.606707 & -0.91247 & 2.60439 \\
\hline 0.7 & -0.87841 & 2.62606 & 33 & 0.5 & -0.878388 & 2.625937 & -0.87498 & 2.60688 \\
\hline 0.9 & -0.84043 & 2.64993 & 33 & 0.5 & -0.840390 & 2.649740 & -0.85964 & 2.62407 \\
\hline
\end{tabular}


Table 7: Comparison between indirect RBFs and other solutions of $F^{\prime \prime}(0)$ and $H^{\prime}(0)$ for some $\phi$ at $\beta=6$ and $\varepsilon=0.1$.

\begin{tabular}{|c|c|c|c|c|c|c|c|c|}
\hline \multirow[b]{2}{*}{$\phi$} & \multicolumn{2}{|c|}{ Runge-Kutta [19] } & \multicolumn{4}{|c|}{ IRBF method } & \multicolumn{2}{|c|}{ HAM [19] } \\
\hline & $F^{\prime \prime}(0)$ & $H^{\prime}(0)$ & $N$ & c & $F^{\prime \prime}(0)$ & $H^{\prime}(0)$ & $F^{\prime \prime}(0)$ & $H^{\prime}(0)$ \\
\hline 1.0 & -1.60501 & 3.85515 & 34 & 0.7 & -1.605015 & 3.855167 & -1.56517 & 3.94163 \\
\hline 0.5 & -1.26561 & 3.18526 & 34 & 0.7 & -1.265601 & 3.185266 & -1.25134 & 3.08218 \\
\hline 0.0 & -0.98358 & 2.57743 & 33 & 0.5 & -0.983591 & 2.577462 & -0.98289 & 2.57162 \\
\hline-0.5 & -0.76447 & 2.06901 & 34 & 0.7 & -0.764451 & 2.068932 & -0.77038 & 2.12820 \\
\hline-1.0 & -0.60299 & 1.67194 & 30 & 0.5 & -0.602973 & 1.671875 & -0.59347 & 1.69706 \\
\hline
\end{tabular}

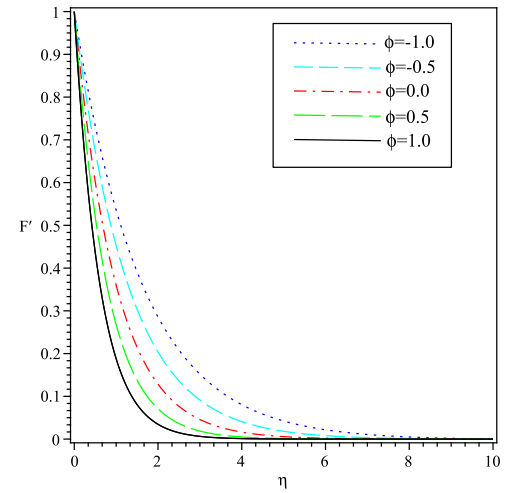

Fig. 6: The profile velocity $F^{\prime}(\eta)$ obtained by the direct RBFs for different values of $\phi$ at $\beta=6$ and $\varepsilon=0.1$.

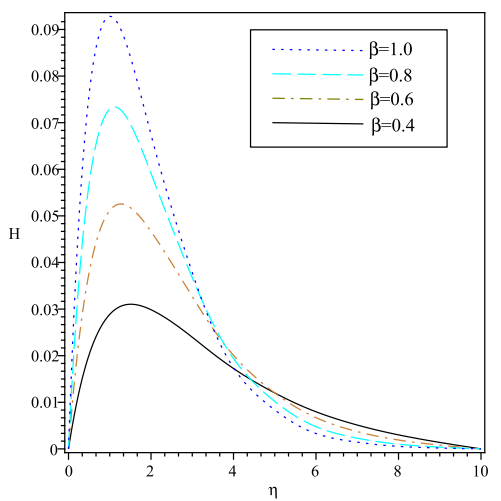

Fig. 7: The profile micro-rotation $H(\eta)$ obtained by the indirect RBFs for different values of $\beta$ at $\phi=0$ and $\varepsilon=0.1$.

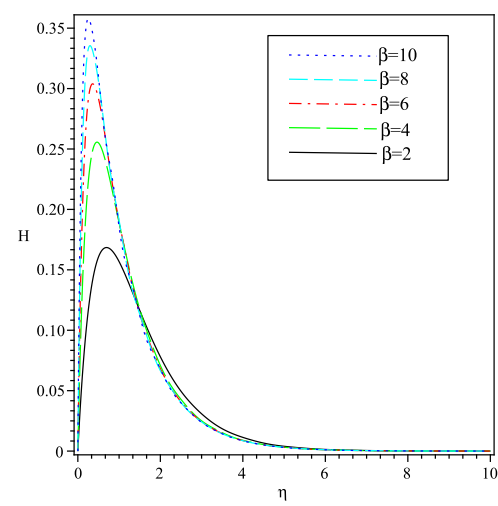

Fig. 8: The profile micro-rotation $H(\eta)$ obtained by the indirect RBFs for different values of $\beta$ at $\phi=0$ and $\varepsilon=0.1$.

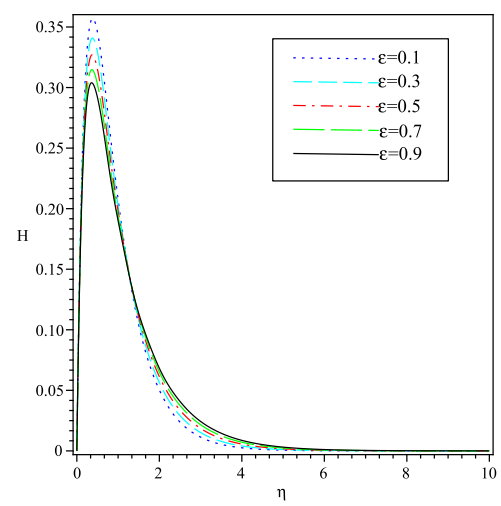

Fig. 9: The profile micro-rotation $H(\eta)$ obtained by the indirect RBFs for different values of $\varepsilon$ at $\beta=6$ and $\phi=0$. 


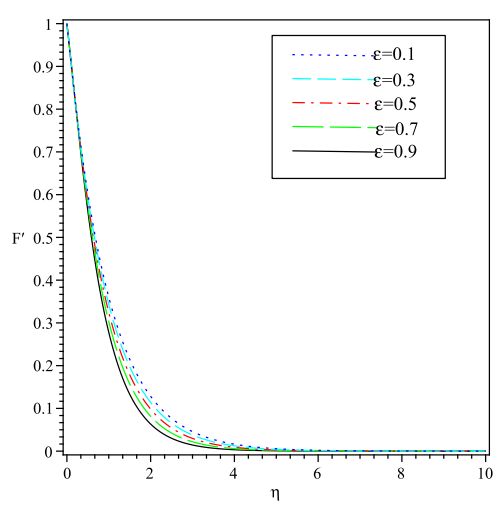

Fig. 10: The profile velocity $F^{\prime}(\eta)$ obtained by the indirect RBFs for different values of $\varepsilon$ at $\beta=6$ and $\phi=0$.

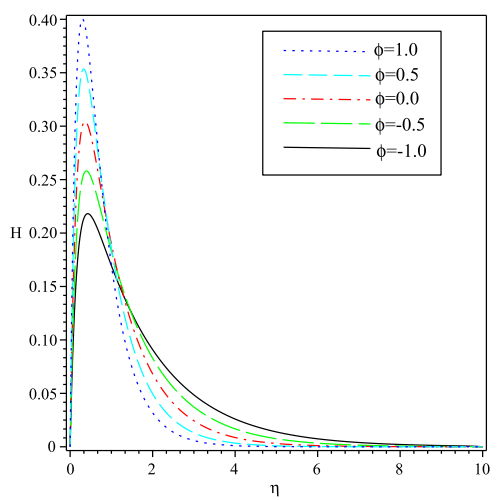

Fig. 11: The profile micro-rotation $H(\eta)$ obtained by the indirect RBFs for different values of $\phi$ at $\beta=6$ and $\varepsilon=0.1$.

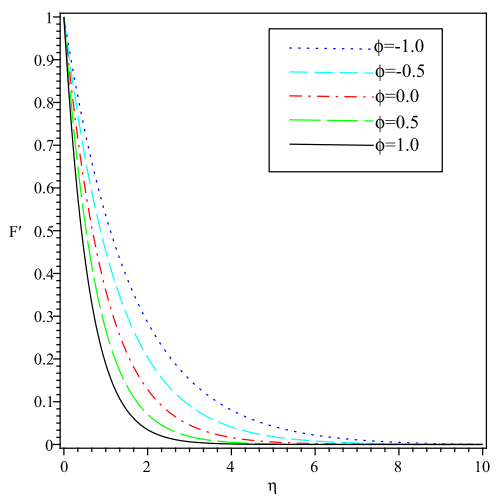

Fig. 12: The profile velocity $F^{\prime}(\eta)$ obtained by the indirect RBFs for different values of $\phi$ at $\beta=6$ and $\varepsilon=0.1$.

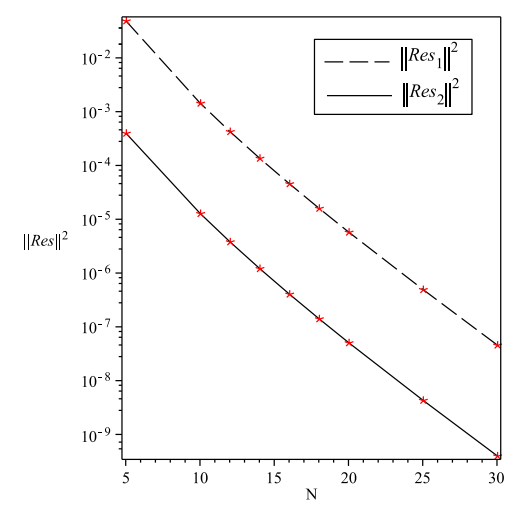

Fig. 13: Graph of $\|$ Res $\|^{2}$ by indirect RBFs solution for $\phi=0, \beta=0.4$ and $\varepsilon=0.1$.

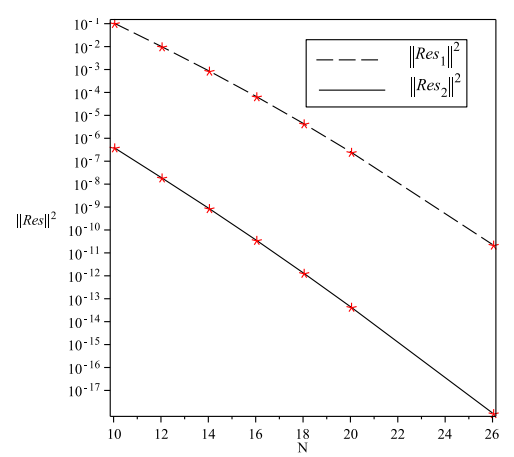

Fig. 14: Graph of $\|\operatorname{Res}\|^{2}$ by direct RBFs solution for $\phi=0, \beta=0.4$ and $\varepsilon=0.1$.

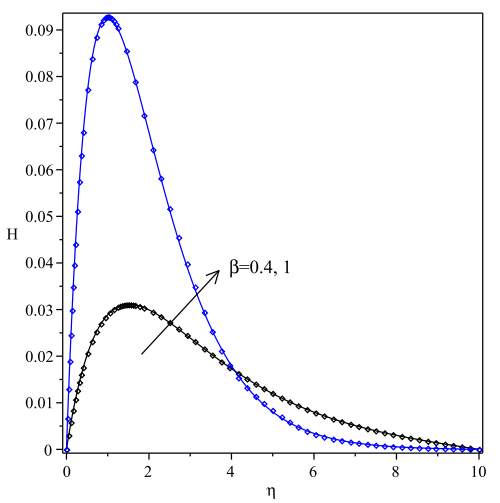

Fig. 15: Comparison between direct (line) and indirect RBFs (points) for $\beta=0.4,1, \phi=0$ and $\varepsilon=0.1$. 
profile $H(\eta)$ increases in the adjacency of the sheet but the reverse happens as one move away from it. The parameter $\beta$ have the few effect on the velocity $F^{\prime}(\eta)$. For this reason, we have not drawn the figure of it.

Figures 3-4 displayed the effect of coupling parameter $\varepsilon$ on micro-rotation $H(\eta)$ and fluid velocity $F^{\prime}(\eta)$. As shown in Figure 3, micro-rotation $H(\eta)$ increases with the increasing values of the coupling parameter $\varepsilon$ near the surface of the sheet. Also in Figure 4 shows that with increase of $\varepsilon$ the value of wall shear stress $\left|F^{\prime \prime}(0)\right|$ decreases near the wall.

Figures 5-6 illustrate the effect of $\phi$ in cases of suction $\phi>0$ and injection $\phi<0$ on micro-rotation $H(\eta)$ and the velocity $F^{\prime}(\eta)$ profile. As shown in Figure 5, by increasing the value of $\phi$, micro-rotation profile $H(\eta)$ increases in the adjacency of the sheet but the reverse happens as one move away from it. Also in Figure 6 displays that velocity profile $F^{\prime}(\eta)$ decreases with increasing the values of $\phi$.

In the case of IRBF method, we discussed the results on $\beta$ in Figures 7-8, on coupling parameter $\varepsilon$ in Figures 910, on $\phi$ in Figures 11-12. Results above are also true for IRBF solution.

The radial basis functions listed in Table 1 contain a shape parameter $c$ that must be specified by the user. But here, by the meaning of residual function, we try to minimize $\|\operatorname{Res}(z)\|^{2}$ by choosing good shape parameter $c$. We define $\|\operatorname{Res}(z)\|^{2}$ as

$$
\|\operatorname{Res}(z)\|^{2}=\int_{0}^{b} \operatorname{Res}^{2}(z) d z \simeq \sum_{j=0}^{m} \omega_{j} \operatorname{Res}^{2}\left(\frac{b}{2} s_{j}+\frac{b}{2}\right),
$$

where

$$
\begin{aligned}
& \omega_{j}=\frac{b}{\left(1-s_{j}^{2}\right)\left(\left.\frac{d}{d s} P_{m+1}(s)\right|_{s=s_{j}}\right)^{2}}, j=0,1, \ldots, m, \\
& P_{m+1}\left(s_{j}\right)=0, j=0,1, \ldots, m,
\end{aligned}
$$

$P_{m+1}(s)$ is $(m+1)$ th-order Legendre polynomial and $b$ is the biggest collocation node. The logarithmic graphs of the $\|\operatorname{Res}(x)\|^{2}$ for IRBF and DRBF at $(\beta=0.4, \varepsilon=$ $0.1, \phi=0$ ) are shown in Figure 13 and Figure 14, respectively. This graphs illustrates the convergence rate of the method. Comparison between DRBF solution and IRBF solution shows that the convergence of the IRBF method is faster, because of using less numbers of collocation points.

Finally, in Figure 15 we compared DRBF and IRBF solutions for two cases, one is for $\beta=0.4, \phi=0$ and $\varepsilon=0.1$ and the other is for $\beta=1, \phi=0$ and $\varepsilon=0.1$

Table 2 shows the DRBF approximations of $F^{\prime \prime}(0)$ and $H^{\prime}(0)$ for some $\beta$ when $\varepsilon=0.1$ and $\phi=0$. This table demonstrates the wall shear stress $F^{\prime \prime}(0)$ decreases with growth of $\beta$ while the values of $H^{\prime}(0)$ increases. Numerical results are compared with Runge-Kutta solution which are obtained by the MATLAB software command ODE45 which is used by [19] and HAM solutions by [19].

Table 3 demonstrates the numerical values of $F^{\prime \prime}(0)$ and $H^{\prime}(0)$ for some values of $\varepsilon$ when $\phi=0$ and $\beta=6$.

Table 4 presents the effects of suction and injection $\phi$ on $F^{\prime \prime}(0)$ and $H^{\prime}(0)$ for $\varepsilon=0.1$ and $\beta=6$. It also shows that suction increases the values of shear stress $F^{\prime \prime}(0)$ at the surface but injection acts exactly in opposition to the suction.

In the case of IRBF method, we discussed the results on $\beta$ in Table 5 , on coupling parameter $\varepsilon$ in Table 6 , on $\phi$ in Table 7 . The results above are also true for IRBF solution.

It should be noted that in this paper we only say that our proposed scheme is numerically more efficient than other methods that are applied to solve this model. Moreover, this model is solved only in the paper [19] using a semi-analytical method named homotopy analysis method (HAM). We can compare the solutions obtained from two meshless methods proposed in this paper with a solution computed in [19]. Bararnia and his/her co-authors [19] proposed a semi-analytical scheme named homotopy analysis method for solving the model. HAM is a multiparameter homotopy method. In fact, in HAM we have some parameters and some auxiliary operators for adjusting the convergence controller. As pointed out by Liao in his book [74], the convergence and rate of approximation for the HAM solution strongly depends on the value of auxiliary parameter $\hbar$, the initial approximation, the auxiliary linear operator, the auxiliary function. However, according to what was reported in [19], the semi-analytical proposed therein allows us to obtain the solution with the error approximately equals $10^{-2}$ using 15th-order. In [19], the authors say no any things about the computer time of algorithm but in the other papers, researchers said that the computer time of homotopy analysis method is too high which is much more than method proposed in this paper.

The main reasons of why the our numerical methods are more efficient than the one proposed by [19] are the following:

(1) In contrast to the proposed meshless methods in this paper, the homotopy analysis method is a iterative scheme so that it needs a lot of iteration numbers. So HAM is complex as pointed out by many researchers.

(2) In HAM, the main disadvantage is that we should suitably choose an initial guess, or infinite iterations are required. In fact, HAM is very much depended on choosing auxiliary parameter which unfortunately has the effect of restricting the application of such methods. Therefore, HAM includes many unknown convergence-control parameters which makes it time consuming for calculating. 
(3) In contrast to the proposed meshless methods in this paper, another disadvantage of these semi-analytical methods such as HAM is the symbolic evaluation of the integrations which can be complex even for a computer algebra package.

(4) HAM gives an approximation to the true solution only in a small region.

(5) In contrast to the proposed meshless methods in this paper, HAM needs too much CPU time to calculate even if the order of approximation is not very high, and thus is often useless in practice even for ordinary differential equations. So it is really hard to implement this semianalytical scheme for solving complex partial differential equation in regular or non-regular domain with a small amount of complexity.

It was shown that the present work provides acceptable approach for incompressible micro-polar fluid in steady two-dimensional motion, driven by a flat sheet that is linearly stretching away from a fixed point. It was also confirmed by logarithmic figures of residual function that this approach has an exponentially convergence rate. Additionally, high convergence rates and good accuracy are obtained by the proposed method using relatively low numbers of data points.

The numerically convergence error of proposed approximated solution is shown in Figures 13 and 14. Paying wise attention to these figures, it can be seen that the residual function decreases, as the number of basis increases. In fact, in Figure 13, we can see when $N=10$, $\|\operatorname{Res}(x)\|_{2} \simeq 10^{-2}$ and when $N=30,\|\operatorname{Res}(x)\|_{2} \simeq 10^{-7}$. It shows fast convergence of the proposed method. Comparison between DRBF solution and IRBF solution in Tables (2)-(7) shows that the convergence of the IRBF method is faster than DRBF, also IRBF in comparison with DRBF has same accuracy while it use less numbers of collocation points.

Paying attention, from these tables and figures we observe that the accuracy grows as the number of nodes increases gradually, this fact can be understood more clearly from the data shown in Figures 13 and 14 since as the number of nodes increases, we find the approximations that possess more correct digits. Then the option price can be computed with a small error in a small computer time. This indicates that the numerical solution converges to the true solution as the number of nodes increases gradually. Again we do confirm that the true solution is not available here. Therefore, we use instead the reference solution which is described in [19], where it has been obtained by performing a simulation named RK method.

\section{Concluding remarks}

Collocation method based on radial basis functions is applied to observe the behavior of micro-polar flow due to a linearly stretching of porous sheet. Here, we proposed two numerical methods for this problem, one is the direct RBFs by using the Multiquadrics (MQ) function and the other is indirect RBFs by using inverse multiquadrics (IMQ) function. In both methods, we have the zeros of the shifted Legendre polynomial as the collocation nodes. Numerical results show that the present IRBF method achieves a high degree of accuracy and high rate of convergence. It was also confirmed by logarithmic figures of residual functions that these approaches have an exponentially convergence rate. Additionally, through the comparison with other methods [19], we have showed that the both RBFs approaches have good reliability and efficiency.

Acknowledgement: The research was supported by a grant from Shahid Beheshti University.

\section{References}

[1] AC. Eringen, Theory of micropolar fluids, New York, Springer, 2001.

[2] AC. Eringen, Microcontinuum field theories: II. Fluent media, J. Math. Mech. 16 (1966) 1-18.

[3] G. Łukaszewicz, Micropolar fluids. Theory and applications, modeling and simulation in science, engineering and technology, Birkhäuser, Boston, 1999.

[4] T. Ariman, M. A. Turk, N.D. Sylvester, Microcontinuum fluid mechanics-a review, Int. J. Eng. Sci. 12 (1974) 273-293.

[5] L. J. Crane, Flow past a stretching plate, Z. angew. Math. Phys., 21 (1970) 645-647.

[6] A. Ishak, R. Nazar, I. Pop, Moving wedge and flat plate in a micropolar fluid, Int. J. Eng. Sci. 44 (2006) 1225-1236.

[7] A. Ishak, R. Nazar, I. Pop, Boundary-layer flow of a micropolar fluid on a continuous moving or fixed surface, Can. J. Phys. 84 (2006) 399-410.

[8] A. Ishak, R. Nazar, I. Pop, Flow of a micropolar fluid on a continuous moving surface, Arch. Mech. 58 (2006) 529-41.

[9] IA. Hassanien, RSR. Gorla, Heat transfer to a micropolar fluid from a nonisothermal stretching sheet with suction and blowing, Acta. Mech. 84 (1990) 191-203.

[10] FM. Hady, On the solution of heat transfer to micropolar fluid from a nonisothermal stretching sheet with injection, Int. J. Numer. Meth. Heat. Fluid. Flow. 6 (1996) 99-104.

[11] PS. Gupta, AS. Gupta, Heat and mass transfer on a stretching sheet with suction or blowing, Can. J. Chem. Eng. 55 (1977) 744-746.

[12] MM. Rahman, MJ. Uddin, A. Aziz, Effects of variable electric conductivity and non-uniform heat source (or sink) on convective micropolar fluid flow along an inclined flat 
plate with surface heat flux, Int. J. Therm. Sci. (2010) doi: 10.1016/j.ijthermalsci.2009.05.003.

[13] MM. Rahman, IA. Eltayeb, SM. Mujibur Rahman, Thermomicropolar fluid flow along a vertical permeable plate with uniform surface heat flux in the presence of heat generation, Therm. Sci. 13 (2009) 23-36.

[14] MM. Rahman, Convective flows of micropolar fluids from radiate isothermal porous surfaces with viscous dissipation and joule heating, Commun. Nonlinear. Sci. Numer. Simul. 14 (2009) 3018-3030.

[15] MM. Rahman, T. Sultana, Radiative heat transfer flow of micropolar fluid with variable heat flux in a porous medium, Nonlinear. Anal. Model. Control. 13 (2008) 71-87.

[16] MM. Rahman, MA. Sattar, Transient convective flow of micropolar fluid past a continuously moving vertical porous plate in the presence of radiation, Int. J. Appl. Mech. Eng. 12 (2007) 497-513.

[17] MM. Rahman, MA. Sattar, MHD convective flow of a micropolar fluid past a continuously moving vertical porous plate in the presence of heat generation/ absorption, ASME J. Heat. Transfer. 128 (2006) 142-152.

[18] T. M. A. El-Mistikawy, Limiting behavior of micropolar flow due to a linearly stretching porous sheet, Eur. J. Mech. B/Fluids 28 (2009) 253-258.

[19] H. Bararnia, E. Ghasemi, G. Domairry, S. Soleimani, Behavior of micro-polar flow due to linear stretching of porous sheet with injection and suction, Adv. Eng. Softw. (2010) doi:10.1016/j.advengsoft.2009.12.007.

[20] R. Franke, Scattered data interpolation: test of some methods, Math. Comput. 38 (1982) 181-200.

[21] E. J. Kansa, Multiquadrics-A scattered data approximation scheme with applications to Computational fluid-dynamicsI surface approximations and partial derivative estimates, Comput. Math. Appl. 19 (1990) 127-145.

[22] E. J. Kansa, Multiquadrics-A scattered data approximation scheme with applications to computational fluid-dynamics-II solutions to parabolic, hyperbolic and elliptic partial differential equations, Comput. Math. Appl. 19 (1990) 147-161.

[23] M. Sharan, E. J. Kansa, S. Gupta, Application of the multiquadric method for numerical solution of elliptic partial differential equations, Appl. Math. Comput. 84 (1997) 275-302.

[24] M. Zerroukat, H. Power, C. S. Chen, A numerical method for heat transfer problems using collocation and radial basis functions, Int. J. Numer. Meth. Eng. 42 (1998) 1263-1278.

[25] N. Mai-Duy, T. Tran-Cong, Numerical solution of differential equations using multiquadric radial basis function networks, Neural Networks 14 (2001) 185-199.

[26] M. Tatari, M. Dehghan, A method for solving partial differential equations via radial basis functions: Application to the heat equation, Eng. Anal. Bound. Elem. 34 (2010) 206-212.

[27] M. Dehghan, A. Shokri, Numerical solution of the nonlinear Klein-Gordon equation using radial basis functions, J. Comput. Appl. Math. 230 (2009) 400-410.

[28] A. Alipanah, M. Dehghan, Numerical solution of the nonlinear Fredholm integral equations by positive definite functions, Appl. Math. Comput. 190 (2007) 1754-1761.

[29] N. Mai-Duy, T. Tran-Cong, RBF interpolation of boundary values in the BEM for heat transfer problems, I. J. Numer. Meth. Heat Fluid Flow 13 (2003) 611 - 632.
[30] N. Mai-Duy, R.I. Tanner, A collocation method based on onedimensional RBF interpolation scheme for solving PDEs, I. J. Numer. Meth. Heat Fluid Flow 17 (2007) 165 - 186.

[31] B. Sarler, J. Perko, C.S. Chen, Radial basis function collocation method solution of natural convection in porous media, I. J. Numer. Meth. Heat Fluid Flow 14 (2004) 187 - 212.

[32] R. Vertnik, B. Sarler, Meshless local radial basis function collocation method for convective-diffusive solid-liquid phase change problems, I. J. Numer. Meth. Heat Fluid Flow 16 (2006) $617-640$.

[33] S. U. Islam, S. Haqb, A. Ali, A meshfree method for the numerical solution of the RLW equation, J. Comput. Appl. Math. 223 (2009) 997-1012.

[34] N. Mai-Duy, Solving high order ordinary differential equations with radial basis function networks, Int. J. Numer. Meth. Eng. 62 (2005) 824-852.

[35] K. Parand, S. Abbasbandy, S. Kazem, A.R. Rezaei, Comparison between two common collocation approaches based on radial basis functions for the case of heat transfer equations arising in porous medium, Commun. Nonlinear. Sci. Numer. Simul. 16 (2011) 1396-1407.

[36] A. J. Khattak, S. I. A.Tirmizi, S. U. Islam, Application of meshfree collocation method to a class of nonlinear partial differential equations, Eng. Anal. Bound. Elem. 33 (2009) 661-667.

[37] M. Dehghan, M. Tatari, Use of radial basis functions for solving the second-order parabolic equation with nonlocal boundary conditions, Numer. Meth. Part. D. E. 24 (2008) 924-938.

[38] M. Dehghan, A. Ghesmati, Solution of the second-order onedimensional hyperbolic telegraph equation by using the dual reciprocity boundary integral equation DRBIE method, Eng. Anal. Bound. Elem. 34 (2010) 51-59.

[39] M. D. Buhmann, Radial basis functions, Acta Numerica (2000) 1-38.

[40] S.A. Sarra, Adaptive radial basis function method for time dependent partial differential equations, Appl. Numer. Math. 54 (2005) 79-94.

[41] M. D. Buhmann, Radial basis functions: theory and implementations, Cambridge University Press, New York, 2004.

[42] H. Wendland, Scattered data approximation, Cambridge University Press, New York, 2005.

[43] A. H. D. Cheng, M. A. Golberg, E. J. Kansa, Q. Zammito, Exponential convergence and $\mathrm{H}-\mathrm{c}$ multiquadric collocation method for partial differential equations, Numer. Meth. Part. D. E. 19 (2003) 571-594.

[44] R. E. Carlson, T. A. Foley, The parameter $R 2$ in multiquadric interpolation, Comput. Math. Appl. 21 (1991) 29-42.

[45] A. E. Tarwater, A parameter study of Hardy's multiquadric method for scattered data interpolation, Report UCRL-53670, Lawrence Livermore National Laboratory, 1985.

[46] G.E. Fasshauer, J.G. Zhang, On choosing "optimal" shape parameters for RBF approximation, Numer Algorithms 45 (2007) 346-368.

[47] N. Mai-Duy, T. Tran-Cong, Numerical solution of Navier-Stokes equations using multiquadric radial basis function networks, Int. J. Numer. Meth. Fl. 37 (2001) 65-86.

[48] W. R. Madych, S. A. Nelson, Multivariate interpolation and conditionally positive definite functions, Approx. Theory Appl. 4 (1988) 77-89.

[49] B. Fornberg, G. Wright, Stable computation of multiquadric interpolants for all values of the shape parameter, Computers 
Math. Applic. 48 (2004) 853-867.

[50] S. A. Sarra, Integrated multiquadric radial basis function approximation methods, Comput. Math. Appl. 51 (2006) 12831296.

[51] M. J. D. Powell, The theory of radial basis function approximation in 1990, Clarendon, Oxford, 1992.

[52] RL. Hardy, Multiquadric equations of topography and other irregular surfaces, J. Geophys. Res. 176 (1971) 1905-1915.

[53] J. Shen, T. Tang, High order numerical methods and algorithms, Chinese Science Press, 2005.

[54] M. A. Golberg, Some recent results and proposals for the use of radial basis functions in the BEM, Eng. Anal. Bound. Elem. 23 (1999) 285-296.

[55] M. Dehghan, A. Shokri, A meshless method for numerical solution of the one-dimensional wave equation with an integral condition using radial basis functions, Numer. Algorithms. 52 (2009) 461-477.

[56] N. Mai-Duy, T. T. Cong, An efficient indirect RBFN-based method for numerical solution of PDEs, Numer. Meth. Part. D. E. 21 (2005) 770-790.

[57] N. Mai-Duy, T. T. Cong, Approximation of function and its derivatives using radial basis function networks, Appl. Math. Modelling. 27 (2003) 197-220.

[58] N. Mai-Duy, T. T. Cong, Solving biharmonic problems with scattered-point discretization using indirect radial-basisfunction networks, Eng. Anal. Bound. Elem. 30 (2006) 77-87.

[59] S. Rippa, An algorithm for selecting a good parameter $\mathrm{c}$ in radial basis function interpolation, Advan. Comp. Math. 11 (1999) 193-210.

[60] J. G. Wang, G. R. Liu, On the optimal shape parameters of radial basis functions used for $2 \mathrm{~d}$ meshless methods, Comput. Meth. Appl. Mech. Eng. 191 (2002) 2611-2630.

[61] L. V. Ballestra, G. Pacelli, Pricing European and American options with two stochastic factors: A highly efficient radial basis function approach, J. Econ. Dyn. Cont. 37 (2013) 1142-1167.

[62] L. V. Ballestra, G. Pacelli, A radial basis function approach to compute the first-passage probability density function in twodimensional jump-diffusion models for financial and other applications, Eng. Anal. Bound. Elem. 36 (2012) 1546-1554.

[63] L. V. Ballestra, G. Pacelli, Computing the survival probability density function in jump-diffusion models: A new approach based on radial basis functions, Eng. Anal. Bound. Elem. 35 (2011) 1075-1084.
[64] J. A. Rad, S. Kazem, M. Shaban, K. Parand, A. Yildirim, Numerical solution of fractional differential equations with a Tau method based on Legendre and Bernstein polynomials, Math. Meth. Appl. Sci. 37 (2014), 329-342.

[65] K. Parand, M. Shahini, M. Dehghan, Rational Legendre pseudospectral approach for solving nonlinear differential equations of Lane-Emden type, J. Comput. Phys. 228 (2009) 88308840 .

[66] K. Parand, Y. Lotfi, J. A. Rad, An accurate numerical analysis of the laminar two-dimensional flow of an incompressible EyringPowell fluid over a linear stretching sheet, Euro. Phys. J. Plus 132 (2017) 397-418.

[67] J. A. Rad, J. Hook, E. Larsson, L. von Sydow, Forward deterministic pricing of options using Gaussian radial basis functions, J. Comput. Sci. 24 (2018) 209-217

[68] J. A. Rad, K. Parand, L. V. Ballestra, Pricing European and American options by radial basis point interpolation, Appl. Math. Comput. 251 (2015) 363-377.

[69] J. A. Rad, K. Rashedi, K. Parand, H. Adibi, The meshfree strong form methods for solving one dimensional inverse CauchyStefan problem, Eng. Comput. 33 (2017) 547-571.

[70] K. Rashedi, H. Adibi, J. A. Rad, K. Parand, Application of meshfree methods for solving the inverse one-dimensional Stefan problem, Eng. Anal. Bound. Elem. 40 (2014) 1-21.

[71] S. Kazem, J. A. Rad, K. Parand, A meshless method on nonFickian flows with mixing length growth in porous media based on radial basis functions: A comparative study, Comput. Math. Appl. 64 (2012) 399-412.

[72] S. Kazem, J. A. Rad, K. Parand, M. Shaban, H. Saberi, The numerical study on the unsteady flow of gas in a semi-infinite porous medium using an RBF collocation method, I. J. Comput. Math. 89 (2012) 2240-2258.

[73] K. Parand, J. A. Rad, Kansa method for the solution of a parabolic equation with an unknown spacewise-dependent coefficient subject to an extra measurement, Comput. Phys. Commun. 184 (2013) 582-595.

[74] S.J. Liao, Beyond Perturbation: Introduction to the Homotopy Analysis Method, Chapman and Hall/CRC Press (2003) Boca Raton. 